University of Texas at El Paso

ScholarWorks@UTEP

$1-2015$

\title{
Inverse problems in theory and practice of measurements and metrology
}

Konstantin K. Semenov

Saint-Petersburg State Polytechnical University, semenov.k.k@gmail.com

Gennadi N. Solopchenko

Saint-Petersburg State Polytechnical University

Vladik Kreinovich

The University of Texas at El Paso, vladik@utep.edu

Follow this and additional works at: https://scholarworks.utep.edu/cs_techrep

Part of the Computer Sciences Commons

Comments:

Technical Report: UTEP-CS-14-18b

Short version published in Proceedings of the International Conference on Advanced

Mathematical and Computational Tools in Metrology and Testing AMTCM'2014, St. Petersburg,

Russia, September 9-12, 2014, Paper 050; full paper published in: Franco Pavese (ed.), Advanced

Mathematical and Computational Tools in Metrology and Testing AMTCM'X, World Scientific,

Singapore, 2015, pp. 330-339.

\section{Recommended Citation}

Semenov, Konstantin K.; Solopchenko, Gennadi N.; and Kreinovich, Vladik, "Inverse problems in theory and practice of measurements and metrology" (2015). Departmental Technical Reports (CS). 824.

https://scholarworks.utep.edu/cs_techrep/824

This Article is brought to you for free and open access by the Computer Science at ScholarWorks@UTEP. It has been accepted for inclusion in Departmental Technical Reports (CS) by an authorized administrator of ScholarWorks@UTEP.For more information, please contact Iweber@utep.edu. 


\title{
INVERSE PROBLEMS IN THEORY AND PRACTICE OF MEASUREMENTS AND METROLOGY
}

\author{
SEMENOV K. K., SOLOPCHENKO G. N. \\ Department of measurement informational technologies, St. Petersburg State \\ Polytechnical University, 29, Polytechnicheskaya str., St. Petersburg, 195251, Russia \\ KREINOVICH V. YA. \\ Department of Computer Science, University of Texas at El Paso, \\ $500 \mathrm{~W}$. University, El Paso, TX 79968, USA
}

\begin{abstract}
In this paper, we consider the role of inverse problems in metrology. We describe general methods of solving inverse problems which are useful in measurements practice. We also discuss how to modify these methods in situations in which there is a need for real-time data processing.
\end{abstract}

\section{Introduction}

What mathematical physics calls inverse problems is, in effect, the class of problems, which are fundamental in measurement theory and practice $[1,2]$. The main objective of such problems is to develop procedures for acquiring information about objects and phenomena, accompanied by decreasing the distortion caused by the measuring instruments. Lord Rayleigh was the first to formulate such problem in 1871, on the example of spectroscopy. His purpose was to maximally decrease the influence of diffraction. Rayleigh showed that in mathematical terms, the problem of reconstructing the actual spectrum $x(v)$ from the measured signal $y(u)$ can be reformulated as the problem of solving an integral equation

$$
y(u)=\int_{-\infty}^{\infty} K(u-v) \cdot x(v) d v,
$$

where $K(u-v)$ is the apparatus function of the spectrometer - which describes the distortion caused by diffraction.

The relation between inverse problems and measurements was emphasized by G. I. Vasilenko [3], who explicitly stated that the main objective of the inverse problem is "restoring the signals" or "reduction to the ideal instrument". 
Eq. (1) is the integral Fredholm's equation of first type; it can be represented in the form $y(u)=\mathbf{A} x(v)$, where $\mathbf{A}$ is a compact linear operator of convolution - which describes a generic analog transformation of a signal inside a measuring instrument - and $K(u-v)$ is the kernel of this operator. From the mathematical viewpoint, the solution of Eq. (1) can be expressed as $x(v)=\mathbf{A}^{-1} y(u)$, where $\mathbf{A}^{-1}$ is the inverse operator to the compact operator $\mathbf{A}$. From the practical viewpoint, however, we have a problem: it is known that such inverse operators are not bounded (see [4, p. 509]); as a result, a small noise in the measured signal can lead to drastic changes in the reconstructed solution $x(v)$. Such problems are known as ill-posed. A general approach of generating a physically reasonable solution to this problem - known as regularization - was formulated by A. N. Tikhonov in 1963 [5].

\section{Inverse problems in metrology}

If we take into account the inaccuracy $e(u)$ with which we register the output signal registration and the inaccuracy $\varepsilon(u-v)$ with which we know the apparatus function of the measurement device, then Eq. (1) will have the form $y(u)=\int_{-\infty}^{\infty} K_{\varepsilon}(u-v) x(v) d v+e(u)$. This equation with infinite (symmetric) integration limits describes spatial distortion processes in spectroscopy, chromatography, and in acoustic and other antenna-based measurements. For dynamic measurements - i.e., for measuring dynamic signals - the measurement result can only depend on the past values of the signal, so integration starts at 0 :

$$
y(t)=\int_{0}^{\infty} K_{\varepsilon}(t-\tau) x(\tau) d \tau+e(t)=\mathbf{A}_{\varepsilon} x(t)+e(t),
$$

where $\mathbf{A}_{\varepsilon}$ is the convolution operator with the kernel $K_{\varepsilon}(t-\tau)$ (known with inaccuracy $\varepsilon(t-\tau))$ and $e(t)$ is the additive noise.

The main idea behind Tikhonov's regularization is that we look for an (approximate) solution $\tilde{x}(t)$ to Eq. (2) by minimizing an appropriate stabilizing functional $\Omega(x(t))$ in Sobolev's space of smooth functions [5]. Usually, a functional $\Omega(x(t))=\beta_{0} \int_{0}^{\infty} \widetilde{x}^{2}(t) d t+\beta_{1} \int_{0}^{\infty}\left[\widetilde{x}^{\prime}(t)\right]^{2} d t, \beta_{0}>0$ and $\beta_{1}>0$, is used on the condition that the difference between $y(t)$ and $\mathbf{A} \widetilde{x}(t)$ is of the same order as the error $\Delta$ caused by $e(t)$ and $\varepsilon(t):\|\mathbf{A} \tilde{x}(t)-y(t)\|^{2}=\Delta^{2}$. The Lagrange multiplier techniques reduces this constrained optimization problem to the unconstrained 
optimization of the functional [5]:

$$
\min _{x(t)}\left[\|\mathbf{A} x(t)-y(t)\|^{2}+\alpha \Omega(x(t))\right],
$$

where $\alpha$ is called a regularization parameter.

\subsection{The minimal modulus principle}

When we have an a priori information about the norm of the solution and/or its derivative, we can find $\alpha$. In particular, we can use fuzzy (imprecise) expert $a$ priori information [6]. In the absence of such a priori information, we can use the principle of minimal modulus $[7,8]$ to select $\alpha$.

This method is based on the fact that in the frequency domain, the stabilizing functional takes the form $\Omega(x(j \omega))=\beta_{0} \int_{0}^{\infty}|x(j \omega)|^{2} d \omega+\beta_{1} \int_{0}^{\infty} \omega^{2}|x(j \omega)|^{2} d \omega$, where $j$ is imaginary unit and $\omega$ is circular frequency. The minimum of this functional is attained when the modulus $|x(j \omega)|$ is minimal.

Fourier transform of Eq. (2) leads to $y(j \omega)=K_{\varepsilon}(j \omega) \cdot x(j \omega)+e(j \omega)$. Based on $95 \%$ confidence intervals $K(\tau)-\varepsilon_{0.95}(\tau) \leq K_{\varepsilon}(\tau) \leq K(\tau)+\varepsilon_{0.95}(\tau)$ and $-e_{0.95}(t) \leq e(t) \leq e_{0.95}(t)$ in time domain, we can find the ellipses describing uncertainty in the frequency domain [9].

As a result, for every frequency $\omega_{i}$ we obtain two error-related ellipses in the complex plane: the first one centered in $y\left(j \omega_{i}\right)$ (Fourier transform of output signal) and another one centered at the value $K_{\varepsilon}\left(j \omega_{i}\right)$ (Fourier transform of apparatus function), as shown on Fig. 1. As shown in [7], for all values $\omega_{i}$ the value $\widetilde{x}\left(j \omega_{i}\right)$ corresponding to the regularized solution is equal to $\widetilde{x}\left(j \omega_{i}\right)=y_{*}\left(j \omega_{i}\right) / K^{*}\left(j \omega_{i}\right)$, where $K^{*}\left(j \omega_{i}\right)$ is point on the ellipse which is

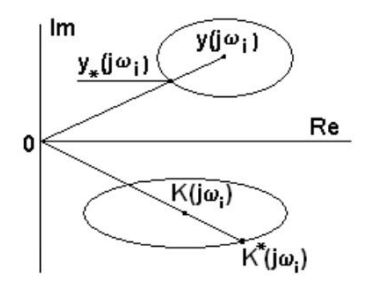

Fig. 1. Illustration of minimal modulus principle the farthest from the coordinates origin, and $y_{*}\left(j \omega_{i}\right)$ is the point on the corresponding ellipse that is the closest to the coordinates origin. This prevents from the situation when there is zero value in denominator. So, the problem stops being incorrect, but numerator $y_{*}\left(j \omega_{i}\right)$ of the ratio $\widetilde{x}\left(j \omega_{i}\right)$ sustains a step to zero value at some frequency. This causes Gibbs phenomenon when we perform inverse Fourier transform of $\widetilde{x}(j \omega)$. In each concrete case, manual adjustment of input data error characteristics may 
decrease effect's influence.

From Fig. 1, it is clear that this solution indeed minimizes the modulus $|x(j \omega)|$, and the condition $\| \mathbf{A} \widetilde{x}(t)-\left.y(t)\right|^{2}=\Delta^{2}$ holds. After applying the inverse Fourier transform to the solution $\widetilde{x}\left(j \omega_{i}\right)$, we get the desired regularized solution to the inverse problem - in other words, we achieve the desired reduction to the ideal measuring instrument. We have shown that this method works very well in many practical situations $[10,11]$. This method also allows us to take into account the "objective" prior information about errors and also "subjective" information - as described by (possibly imprecise) expert estimates [6].

\subsection{The inverse filter}

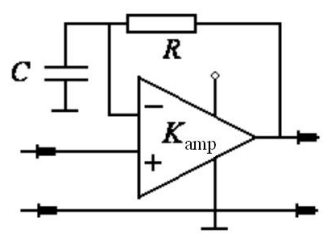

Fig. 2. Inverse filter circuit

The principle of minimal modulus can only be used after the whole signal is measured. This is reasonable in spectroscopy and chromatography, but in processing dynamic signals, we often need to produce results in real time, before all the measurements are finished. This can be achieved by using an inverse filter, which can be physically implemented as one or several sequential dynamically stable circuits. An example is given on Fig. 2. If the amplifier gain is $K_{\text {amp }}$ and $R$ and $C$ are the resistance and capacitance of inertial $R C$-circuit, then the complex frequency characteristic (CFC) of circuit on Fig. 2 is equal to

$$
K_{f}(j \omega)=\frac{K_{\mathrm{amp}}}{1+K_{\mathrm{amp}}} \cdot(1+j \omega R C) \cdot\left[1+j \omega \cdot \frac{R C}{1+K_{\mathrm{amp}}}\right]^{-1} .
$$

This filter can be used if the modulus of CFC of the measuring instrument is monotonically decreasing. For example, such property is usual for thermistors, thermocouples, Hall sensors for current strength etc. Such gauges have first order CFC:

$$
K_{g}(j \omega)=\frac{K_{0}}{1+j \omega \tau_{\mathrm{g}}},
$$

where $\tau_{\mathrm{g}}$ is its time constant and $K_{0}$ is gain coefficient for static mode. In this case, if values of $R$ and $C$ for inverse filter (Fig. 2) are such that $R C=\tau_{\mathrm{g}}$, then series of the gauge and the inverse filter placed after it will have $\mathrm{CFC}$ equal to

$$
K_{g}(j \omega) \cdot K_{f}(j \omega)=\frac{K_{0} \cdot K_{\mathrm{amp}}}{1+K_{\mathrm{amp}}} \cdot\left[1+j \omega \cdot \frac{R C}{1+K_{\mathrm{amp}}}\right]^{-1} .
$$

We can see that time constant of such series is decreased in $\left(1+K_{\text {amp }}\right)>>1$ 
times versus $\tau_{\mathrm{g}}$. This causes corresponded response acceleration with the same ratio and represents the solution for inverse problem of signal restoration.

If the order of CFC for the measurement device is larger than one, then the quantity of first-order inverse filters (Fig. 2) that should be concatenated one after another is the same as the order value. The positive result can be achieved with individual tuning of gain and parameters $R$ and $C$ for every first-order circuit. The inverse problem solution can be achieved using the similar inverse filters even for convertors witch CFC order cannot be rated.

Let us examine the example of using such inverse filter for $\Sigma \Delta-$ AnalogDigital Conversion: let us consider approximation of frequency characteristic for ADC ADS1256 [12]. This ADC is used for digitizing analog signals with frequency bands $(0 \div 25),(0 \div 50)$ and $(0 \div 500) \mathrm{Hz}$. To construct inverse filter to improve its metrological properties, we should use fractionally rational approximation to $\mathrm{ADC}$ frequency characteristics.

To approximate $\operatorname{ADS} 1256 \mathrm{CFC} K_{\mathrm{ADC}}(j \omega)$, we can use separate values of its squared amplitude frequency characteristic (AFC) $\mid K_{\mathrm{ADC}}(j \omega)^{2}$ that is presented by ADC producer [12]:

$$
\left|K_{A D C}(j \bar{\omega})\right|=\left|\frac{\sin (\pi \cdot \bar{\omega})}{64 \cdot \sin (\pi \cdot \bar{\omega} / 64)}\right|^{5} \cdot\left|\frac{\sin (\pi \cdot \bar{\omega} \cdot N)}{N \cdot \sin (\pi \cdot \bar{\omega})}\right|,
$$

where $\bar{\omega}=\omega / \omega_{s}=f / f_{s}$ is relative frequency, $f$ is ADC input signal frequency, $f_{s}=30 \mathrm{kHz}$ is ADC maximum sampling frequency, $N$ is quantity of averaging output values, $\omega=2 \pi f$ and $\omega_{s}=2 \pi f_{s}$ are angular frequencies.

The mentioned data points are placed in the second row of table 1 . Fractionally rational approximation was performed for function $\left|K_{\mathrm{ADC}}(j \omega)\right|^{2}$. This function is real-valued, its argument is $\omega^{2}$. So, we can apply traditional approximation techniques that are developed for real-valued functions.

Two variants of approximation were considered: the case when $N=1$ and frequency band is $[0,0.06666]$ for $\bar{\omega}$ for approximation and $[0,2000] \mathrm{Hz}$ for $f$ and the case when $N=8$ and band is $[0,0.06]$ for $\bar{\omega}$ or $[0,1800] \mathrm{Hz}$ for $f$. We use uniform meshes of 81 points for both of cases $N=1$ and $N=8$.

The obtained approximations were factorized to get expression for CFC $K_{\mathrm{ADC}}(j \omega)$. The used factorization method is described in [13]. Approximation accuracy was set to $0.3 \%$. As a result, CFC and phase frequency characteristics $\varphi_{\mathrm{ADC}}(j \bar{\omega})(\mathrm{PFC})$ were obtained:

$$
N=1: \quad \widetilde{K}_{\mathrm{ADC}}(j \bar{\omega})=\frac{1}{1+4.112 \cdot j \bar{\omega}} \text { and } \widetilde{\varphi}_{\mathrm{ADC}}(\bar{\omega})=-\operatorname{arctg}(4.112 \cdot \bar{\omega}) .
$$




$$
\begin{gathered}
N=8: \quad \widetilde{K}_{\mathrm{ADC}}(j \bar{\omega})=\frac{1}{1+2 \cdot 0.8598 \cdot 14.73 \cdot j \bar{\omega}-(14.73 \cdot \bar{\omega})^{2}} \text { and } \\
\widetilde{\varphi}_{\mathrm{ADC}}(\bar{\omega})=-\operatorname{arctg} \frac{25.330 \cdot \bar{\omega}}{1-217.0 \cdot \bar{\omega}^{2}} .
\end{gathered}
$$

In table 1 placed below, values of real $\mathrm{AFC}\left|K_{\mathrm{ADC}}(j f)\right|$ and its obtained approximation $\left|\widetilde{K}_{\mathrm{ADC}}(j f)\right|$ are presented for $N=1$. Fig. 3 illustrates data presented in Table 1. Averaging, that takes a place during analog-to-digital conversation, causes this effect of AFC decreasing.

Table 1. Results of approximation of $K_{\mathrm{ADC}}(j f)$ for ADC ADS1256 $(N=1)$

\begin{tabular}{|l|c|c|c|c|c|c|c|c|c|}
\hline$f, \mathrm{~Hz}$ & 0.0 & 50 & 100 & 200 & 300 & 400 & 500 & 1000 & 2000 \\
\hline$\left|K_{\mathrm{ADC}}(j f)\right|$ & 1.0 & 0.99997 & 0.999908 & 0.99963 & 0.99918 & 0.99854 & 0.9977 & 0.9909 & 0.9640 \\
\hline$\left|\widetilde{K}_{\mathrm{ADC}}(j f)\right|$ & 1.0 & 0.99997 & 0.999906 & 0.99962 & 0.99915 & 0.99850 & 0.9976 & 0.9907 & 0.9644 \\
\hline$\widetilde{\varphi}_{\mathrm{ADC}}(j f)$ & $0.0^{\circ}$ & $-0.40^{\circ}$ & $-0.78^{\circ}$ & $-1.57^{\circ}$ & $-2.35^{\circ}$ & $-3.14^{\circ}$ & $-3.92^{\circ}$ & $-7.8^{\circ}$ & $-15.3^{\circ}$ \\
\hline
\end{tabular}

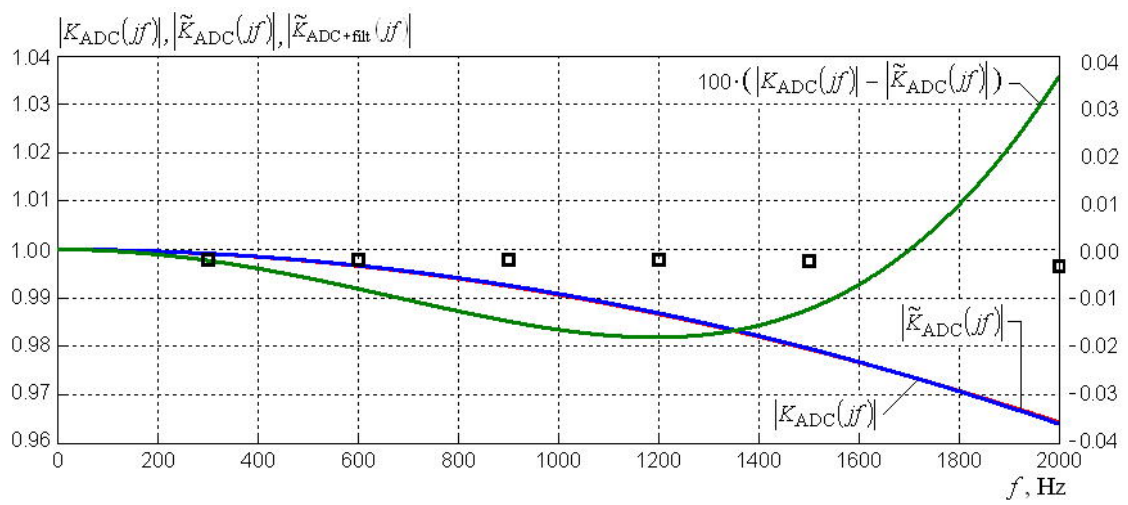

Fig. 3. Results of AFC approximation for ADC ADS1256 (case $N=1$ )

In table 2 the results are placed for the case $N=8$. Fig. 4 contains graphical representation of data from this table.

Table 2. Results of approximation of $K_{\mathrm{ADC}}(j f)$ for ADC ADS1256 $(N=8)$

\begin{tabular}{|c|c|c|c|c|c|c|c|}
\hline$f, \mathrm{~Hz}$ & 0.0 & 300 & 600 & 900 & 1200 & 1500 & 1800 \\
\hline$\left|K_{\mathrm{ADC}}(j f)\right|$ & 1.0 & 0.9888 & 0.9559 & 0.9025 & 0.8310 & 0.7444 & 0.6463 \\
\hline$\left|\widetilde{K}_{\mathrm{ADC}}(j f)\right|$ & 1.0 & 0.9895 & 0.9575 & 0.9035 & 0.8296 & 0.7426 & 0.6512 \\
\hline
\end{tabular}




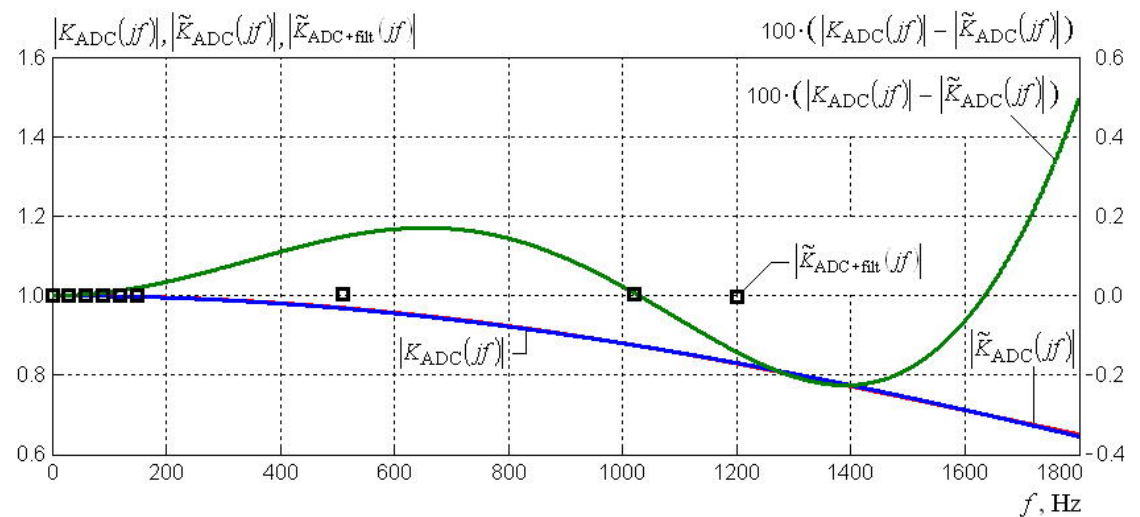

Fig. 4. Results of AFC approximation for ADC ADS1256 (case $N=8$ )

We can see that for frequencies $f$ less than $100 \mathrm{~Hz}$ the AFC is close to unit value (difference is less than $0.02 \%$ ) and PCF is moved for less than $1^{\circ}$ for both of approximations that is acceptable. For frequencies from 500 to $1000 \mathrm{~Hz}$, the AFC differs from 1.0 with error less than $0.5 \%$. Our purpose is now to construct physically realizable inverse filter that will describe obtained approximation.

Let us take into consideration case $N=1$.

The CFC approximation has the first order. So, we can use the simplest inverse filter as presented on Fig. 2. The best AFC correction will be obtained if we choose amplifier's gain equal to $K_{\mathrm{amp}}=500$ and time constant for filter in feedback circuit equal to $\tau_{f}=R \cdot C=21.5 \mu$ s. Let $\left|\widetilde{K}_{\text {filt }}(j f)\right|$ be AFC of inverse filter. Then, the AFC of inverse filter (first circuit in sequence) and ADC (second circuit in sequence) connection will be $\left|\widetilde{K}_{\mathrm{ADC}+\text { filt }}(j f)\right|=\left|\widetilde{K}_{\text {filt }}(j f)\right| \cdot\left|K_{\mathrm{ADC}}(j f)\right|$.

Values of corrected AFC for some frequencies are presented in Table 3 and put on Fig. 3 (marked as squares).

Table 3. Correction with the inverse filter for $\operatorname{ADC} \operatorname{ADS} 1256(N=1)$

\begin{tabular}{|c|c|c|c|c|c|c|}
\hline$f, \mathrm{~Hz}$ & 300 & 600 & 900 & 1200 & 1500 & 2000 \\
\hline$\left|K_{\mathrm{ADC}}(j f)\right|$ & 0.9992 & 0.9967 & 0.9926 & 0.9869 & 0.9796 & 0.9640 \\
\hline$\left|\widetilde{K}_{\mathrm{ADC}+\mathrm{filt}}(j f)\right|$ & 0.9980 & 0.9980 & 0.9979 & 0.9978 & 0.9976 & 0.9971 \\
\hline
\end{tabular}

We see that corrected AFC has essentially wider frequency band.

Let us now take into consideration case $N=8$.

The simplest realization of inverse filter for correction of second-order CFC that is used to describe ADC time-frequency characteristics is concatenation of two inverse filters of first order. Time constant $\tau_{f}$ for each of them should be 
about $\tau_{f}=(3.2 \div 3.6) / f_{s}$ for $\widetilde{K}_{1}(\bar{\omega})$ and $\widetilde{K}_{2}(\bar{\omega})$. Block-scheme of such complex inverse filter is presented on Fig. 5.

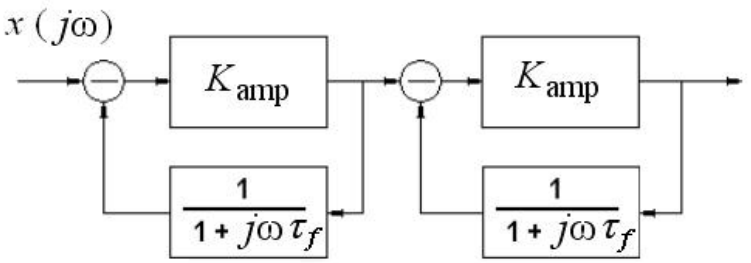

Fig. 5. Block-scheme for inverse filter of second order

Filter of such structure can be easily realized in analog or in digital form. But to obtain higher accuracy, it is better to put inverse filter before ADC and combine it with input gain amplifier.

Time constant $\tau_{f}$ for each of first-order inverse filter on the Fig. 5 should be adjusted using filter mathematical model. It can happen that the best result will be when time constants will be different for these filters. Mathematical modeling can help to determine the best gain value $K_{\mathrm{amp}}$ for direct circuits on Fig. 5. They should have work frequency band wider than frequency diapason that is chosen for CFC correction.

Mathematical modeling shows that for case $N=8$ the satisfactory correction can be achieved if we use two inverse filter of first order, which parameters are $K_{\mathrm{amp}}=100, \tau_{f}=15 \mu \mathrm{s}$ and $K_{\mathrm{amp}}=20, \tau_{f}=6 \mu$ s. Results of such correction are presented in Table 4 and put on Fig. 4 (marked as squares).

Table 4. Correction with the inverse filter for ADC ADS1256 $(N=8)$

\begin{tabular}{|c|c|c|c|c|c|c|c|c|}
\hline$f, \mathrm{~Hz}$ & 30 & 60 & 90 & 120 & 150 & 510 & 1020 & 1200 \\
\hline$\left|\widetilde{K}_{\mathrm{ADC}+\text { filt }}(j f)\right|$ & 1.00002 & 1.00008 & 1.00018 & 1.00032 & 1.0005 & 1.0041 & 1.0050 & 0.9959 \\
\hline$\widetilde{\varphi}_{\mathrm{ADC}+\text { filt }}(j f)$ & $-0.24^{\circ}$ & $-0.48^{\circ}$ & $-0.72^{\circ}$ & $-0.96^{\circ}$ & $-1.2^{\circ}$ & $-5.05^{\circ}$ & $-9.72^{\circ}$ & $-11.8^{\circ}$ \\
\hline
\end{tabular}

We can see that AFC unevenness for frequency band $[0,1] \mathrm{kHz}$ is less than $0.5 \%$ and $\mathrm{PFC}$ has maximal deviation from linearity equal to $1^{\circ}$.

We can conclude that described technique of inverse filter design allows obtaining measuring channel with wider frequency band and faster response. Such technique can be applied to any measurement instrument or converter with monotonically decreased amplitude frequency characteristics.

\section{References}

1. G. Solopchenko, Measurement Techniques. 17 (1974).

2. V. Knorring and G. Solopchenko, Measurement Techniques. 46, 546 (2003). 
3. G. Vasilenko, Theory of signal restoration. (1979). In Russian.

4. S. Mikhlin, Mathematical physics: an advanced course. (1970).

5. A. Tikhonov and V. Arsenin, Solutions of Ill-Posed Problems. (1977).

6. V. Kreinovich, C.-C. Chang, L. Reznik and G. Solopchenko, NASA Conference Publication (NAFIPS-92), 2, 418. (1992).

7. G. Solopchenko. Measurement Techniques, 44, 546 (2001).

8. N. Seregina and G. Solopchenko, Izvestiya AN SSSR. Technical cybernetics, 2, 166 (1984).

9. K. Semenov and G. Solopchenko. Measurement Techniques. 53, 592 (2010). 10. K. Savkov, N. Seregina. Journal of Advanced Materials, 1 (2), 205 (1994). 11. N. Seregina, G. Solopchenko. Pribory i sistemy upravleniya. (4), 19 (1992) In Russian.

12. ADS1255, ADS1256: Very Low Noise, 24-bit Analog-to-Digital Converter. Texas Instruments technical document SBAS288K. Available at: www.ti.com/lit/ds/sbas288k/sbas288k.pdf.

13. V. Kreinovich, G. Solopchenko. Measurement Techniques. 36 (9), 968 (1993). 